FINANÇAS 


\title{
HIGH FREQUENCY TRADING: ANÁLISE DE RETORNO, VOLUME E VOLATILIDADE
}

\author{
HIGH FREQUENCY TRADING: RETURN, VOLUME AND VOLATILITY ANALYSIS
}

Alcides Carlos de Araújo

Universidade de São Paulo

Alessandra Montini

Universidade de São Paulo

\author{
Data de submissão: 09 jul. 2017 . Data de aprovação: \\ 12 dez. 20 17. Sistema de avaliação: Double blind review. \\ Universidade FUMEC / FACE. Prof. Dr. Henrique Cordeiro \\ Martins. Prof. Dr. Cid Gonçalves Filho.
}

\section{RESUMO}

Este artigo tem por objetivo analisar a relação entre volatilidade dos preços e volume na bolsa de valores brasileira em momentos anteriores e posteriores à aplicação das estratégias HFT. Para estudar a relação entre as variáveis, buscou-se comparar os momentos do mercado brasileiro antes HFT e pós HFT. O período antes HFT foi compreendido entre 2000 a 2005; o período pós HFT entre os anos 2009 a 2014. Para estimativa dos parâmetros utilizou-se o método da máxima verossimilhança com informação completa (MV); o método de regressão em 3 estágios (3SLS); o método dos momentos generalizados (GMM) e o modelo $\mathrm{GARCH}(\mathrm{I}, \mathrm{I})$ adotando para otimização o método de Marquadt. Quanto aos resultados, verificou-se que o número de negociações influencia o volume transacionado e, com o crescimento das operações HFT, esta influência apresentou ainda mais força. Quanto à relação entre volatilidade e volume, os coeficientes foram positivos e significativos, sendo que a força da relação tendeu a aumentar a medida que o número de negociações aumentou. $\mathrm{O}$ artigo contribuiu ao demonstrar que a hipótese de distribuição mista (Mixture of Distribution Hypothesis - MDH) está adequada ao mercado brasileiro. Além de trazer um alerta aos operadores de mercado, o uso exagerado do HFT aumentará o volume transacionado, causando mais volatilidade no mercado.

\section{PALAVRAS-CHAVE}

Negociações em alta frequência. Preço-Volume. Fluxo de Informações no Mercado. Volatilidade. 


\section{ABSTRACT}

This paper aims to analyze the relationship between volatility and volume in the Brazilian market exchange in the periods before and after HFT started. The periods before and after HFT were compared to study the relationship between the variables. The period before HFT was between the years 2000 and 2005, and the after HFT period was between years 2009 and 2014. The parameters were estimated by the method of Full Information Maximum Likelihood (MV), the Three-Stage Least Squares (3SLS), the Generalized Method of Moments (GMM) and the GARCH(I,I) model with Marquadt method for optimization. Regarding results, the evaluation showed that the number of negotiations affects the financial volume and this relationship had more impact when HFT activity increased. Regarding the relationship between volatility and volume, the coefficients were positive and significant; the relationship was more impactful when the number of negotiations increased. This article contributes to demonstrate that the Mixture of Distribution Hypothesis - MDH is appropriate for the Brazilian market exchange. Also, it brings a warning to the high frequency traders, the excessive use of the HFT will increase the financial volume, but it could cause more market volatility.

\section{KEYWORDS}

High Frequency Trading. Price-Volume. Market Information Flow. Volatility.

\section{INTRODUÇÃO}

As operações por alta frequência (High Frequency Trading - HFT) cresceram significativamente nos E.U.A. Conforme apresenta Aldridge (20I3), no entanto, ainda estão em fase inicial. Segundo o autor, essas operações representam em média $25 \%$ de toda atividade de negociações no mercado americano, sendo transacionados majoritariamente derivativos de mini-futuros.

Os retornos também não decepcionaram os investidores; em dados apresentados por Aldridge (20I0), alguns fundos HFT (i.e. Medallion Fund) apresentaram rendimentos anuais médios de $35 \%$ (entre 2000 e 2010); os fundos da Renaissance chegaram a superar o S\&P500 em meio à crise de 2008, com rendimentos em torno de $4 \%$ a $6 \%$.

Em pesquisas a respeito do tema, os pesquisadores apresentavam variados pontos positivos a respeito do HFT. Em Aldridge (20I0) são citados quatro pontos benéficos aos mercados quanto à utilização das operações em alta frequência: aumento da eficiência, aumento da liquidez, maior desenvolvimento tecnológico e estabilização dos sistemas de mercado.

Por meio destas evidências, o mercado americano demonstrava sentimento positivo em relação ao HFT. Contudo, o evento ocorrido no dia 6 de Maio de 2010, conhe- 
cido como Flash Crash Day, levantou um debate a respeito da estrutura e estabilidade do mercado financeiro dos E.U.A. no que se referia ao uso dessas operações. Conforme Kirilenko et al. (20I I), naquele dia específico os índices de ações, futuros, opções e fundos do mercado financeiro americano apresentaram uma súbita queda de preços superior a $5 \%$ em um período de 30 minutos, seguida por outra súbita recuperação.

Argumentos em oposição passaram a ser apresentados: os computadores não possuíam, afinal, conhecimento sobre a natureza das empresas ou o que elas fazem; os investidores de HFT seriam capazes de manipular o mercado graças ao rápido ajuste a novas informações e altíssimos volumes financeiros de transação. Este comportamento poderia gerar o acionamento de forma descontrolada de ordens start (entrada) ou stop (saída) alterando o equilíbrio do mercado e aumentando a volatilidade.

Quanto aos retornos, conforme apresenta Seabra (20I4), os fundos nos E.U.A. que utilizam estratégias baseadas em algoritmos de alta frequência estão exibindo desempenho abaixo do esperado nos últimos anos. Como resultado, os cotistas passaram a realizar saques e os fundos perderam participação no mercado.

Depois de um rápido crescimento, as operações por HFT passam por súbita desconfiança entre investidores, analistas e gestores de carteiras - indaga-se até mesmo quantos investidores compreendem o funcionamento dos modelos de alta frequência, conforme cita Kupfer (20II). O próprio órgão regulador do mercado de capitais americano, a SEC (Securities and Exchange Commission), apresentou um documento contendo em torno de 215 perguntas a respeito do funcionamento e regulação da estrutura do mercado financeiro norte americano na presença de operações de alta frequência (SEC, 20I0).

Em busca de respostas para o debate levantado, a principal associação mundial para regulação de valores mobiliários International Organization of Securities Commissions (IOSCO) evidenciou como principais temas de atuação em 2013 a regulação dos negócios de alta frequência, o impacto da tecnologia e a análise destas infraestruturas de mercado (IOSCO, 20I2).

Em relação ao Brasil, Araújo e Montini (2013) apresentaram questões relevantes sobre o tema: "faria sentido este debate no Brasil com mercado ainda em crescimento?" e "as estratégias de HFT apresentariam rentabilidades comparáveis com os retornos vistos nos EUA quando iniciaram estas operações?".

Quanto ao crescimento, existe um esforço pela CVM (Comissão de Valores Mobiliários) para fomentar essas operações. O ritmo de desenvolvimento, porém, não é tão rápido como observado nos E.U.A. (Portugal, 20I0). Uma explicação para o desenvolvimento mais lento foi a morosidade para a completa automação das operações, ocorrida somente em 2005. A partir daquele ano, todas as operações passaram a acontecer de forma eletrônica, encerrando-se também o pregão viva-voz.

Quanto aos retornos, Seabra (20l4) faz referência a uma pesquisa realizada pela FGV, demonstrando que a utilização de algoritmos complexos não garante ao fundo uma melhor performance em comparação com os demais. Do ponto de visto estatístico, as diferenças entre os retornos dos fundos não foram significativas. Do ponto de vista financeiro, as carteiras administradas sem o uso de algoritmos apresentaram 
retorno em torno de $5 \%$ acima das carteiras geridas com base em algoritmos.

Esse resultado era previsto por pesquisadores da área, conforme Seabra (2014), em entrevista com especialistas do mercado financeiro, os modelos funcionam mantendo certas variáveis analisadas estáveis, porém o mercado brasileiro é "pequeno, pouco profundo e muito volátil”. Diante deste fato, seria necessário um número maior de participantes comprando e vendendo em alta velocidade para que o volume transacionado pudesse trazer algum retorno significativo à estratégia utilizada.

Conforme apresentaram os especialistas, o retorno das estratégias por HFT abaixo do esperado está relacionado ao baixo volume de transações na bolsa brasileira. De acordo com Rocha (20I3), existe uma estagnação desde 2008 no volume negociado no mercado financeiro brasileiro.

Mesmo diante do evidente problema relacionado ao volume negociado, empresários brasileiros são otimistas em relação ao HFT. Conforme demonstrou Portugal (2010) os executivos desejam aumentar o número de operações por alta frequência graças à melhor oferta de infraestrutura tecnológica na indústria de investimentos.

Em Mazzoni (20I2) os executivos entrevistados também demonstraram otimismo para com as operações de alta frequência no Brasil, como se constata em afirmações como: "The Brazilian market is in excellent position for growth and our goal is to focus on Brazil". Conforme o autor, empreendedores da área percebem no Brasil um estágio semelhante ao dos E.U.A. antes do rápido crescimento das operações HFT.

O HFT está relacionado com aumentos significativos nos volumes transacionados nas bolsas de valores, alinhando-se ao discutido na análise entre retorno, volume e número de negociações em Araújo e Montini (2013). Diante disto, justifica-se uma pesquisa capaz de observar o relacionamento entre volatilidade do mercado de ações e o volume das transações realizadas, principalmente em um contexto de mercado com presença e crescimento do HFT como o Brasil.

O ambiente de microestrutura do HFT foi estudado no artigo de Araújo e Montini (20l3), em que se buscou observar as relações entre retornos, volume e negociações em momentos antes e pós-iniciação das estratégias HFT à luz das hipóteses clássicas propostas por Karpoff (1987). O principal foco de análise foi a comparação entre os resultados encontrados por Medeiros e Doornik (2008) e adição da variável "número de negociações realizadas" aos modelos.

Os autores encontraram evidências para afirmar que havia associação entre variação absoluta de preços e volume, bem como sua assimetria (retornos positivos necessitam de maior volume transacionado) no período entre os anos de 2000 a 2005. No período com a presença do HFT (anos entre 2009 a 201 3), os coeficientes não foram significativos - o volume transacionado ficou estagnado naquele período.

$O$ artigo contribuiu a literatura por apresentar uma discussão a respeito de um tema ainda pouco discutido no Brasil, porém com significativa demanda por informações. Como limitação, as pesquisas foram restritas à observação das análises de variação absoluta e variação pura dos preços em relação ao volume e número de negociações. Entretanto, a relação entre volume e volatilidade não foi verificada.

Conforme Karpoff (1987) analisar a 
volatilidade é relevante porque a relação entre preço e volume tende a ser mais significativa em mercados em que o fluxo de informação é mais volátil. Diante disto, a força da relação tende a aumentar conforme o número de negociações. Karpoff (1987) também ressalta que o volume é uma variável utilizada para observar o fluxo de informações; deste modo, dado um volume alto em algum período, verifica-se maior variância nos preços.

Mediante tais justificativas, o objetivo do presente artigo é analisar a relação entre a variância das mudanças de preços e volume na bolsa de valores brasileira em momentos antes e pós-iniciação das estratégias HFT. Os próximos tópicos são organizados por revisão da literatura, metodologia, discussões dos resultados e considerações finais.

\section{REVISÃO DA LITERATURA}

Para que seja possível comparar volume transacionado e volatilidade dos preços em ambientes sem e com HFT, torna-se importante definir a expressão High Frequency Trading (HFT). Conforme Aldridge (20I3) na conceituação do termo é importante diferenciá-lo dos conceitos de Electronic Trading e Algorithmic Trading (AT).

O Trading Eletrônico se refere à capacidade de transmissão de ordens eletronicamente, ou seja, sem uso de telefone, carta ou viva-voz. Conforme Aldridge (20I3), as atividades de AT e HFT são subgrupos do Trading Eletrônico; percebe-se na literatura maior ênfase na diferenciação entre AT e HFT.

Aldridge (20I3) e Vuorenmaa (20/3) apresentam conceituação similar: ambos classificam AT e HFT como negociações sistematizadas e automatizadas. Deste modo, as decisões e envio de ordens de negociação ocorrem por meio de programas de computador que analisam os dados e enviam as ordens, funcionando por dias, semanas ou meses. Sistemas assim, porém, podem ser ATs ou HFTs.

Um sistema desenvolvido para AT possui objetivo de minimizar custos de execução, buscando enviar as ordens de negociação quando variáveis como tempo de execução e tamanho do lote estiverem otimizadas. Deste modo, negociações baseadas em AT podem ou não ser executadas imediatamente.

Um sistema desenvolvido para HFT utiliza algoritmos que gerem sinais de negociação e otimizem custos de execução. Conforme Vuorenmaa (20I3), os HFTs utilizam estratégias de negociação que maximizam a rentabilidade, sendo que o tempo de entrada e saída da operação pode levar minutos, segundos e até milissegundos.

Aldridge (20/3) apresenta cinco características associadas ao HFT:

I. Toda operação que utiliza algoritmos de rápida execução.

2. Tecnologia de agilidade: geração de sinais, validação de modelos e execução em tempo supersônico.

3. Frequência de negociação em microssegundos.

4. Alto volume negociado com lotes de tamanho pequeno.

5. Atividade de negociação impraticável por humanos - execução de 200 ordens por segundo, por exemplo.

Diante disto, a característica distintiva do HFT é a duração da posição (comprada ou vendida) sendo no máximo de um dia e sem manutenção de posição para o período subsequente (Aldridge, 20I3).

Quanto à análise entre retornos, vo- 
latilidades dos retornos e volumes nos mercados financeiros, existe uma vasta literatura sobre o tema. Alguns trabalhos relevantes foram originados na década de 50; o tema só recebeu ênfase significativa na década de 70, na formação dos mercados de opções. Nos últimos anos, voltou a ser bastante discutido graças ao crescimento das operações de alta frequência nos E.U.A.

Conforme apresenta Portnoy (20I I), os negociadores que utilizam algoritmos de alta frequência podem alterar o equilíbrio de oferta e demanda. Uma vez que esses modelos podem enviar altas quantidades de ordens com volumes significativos, por meio disto, outros investidores podem seguir a tendência e enviar ainda mais ordens de compra ou venda, alterando os preços significativamente, ainda que de forma completamente artificial.

Nos próximos tópicos apresentaremos resultados de pesquisas visando analisar a relação entre variações de preços e volume negociado $(\Delta \mathrm{peV})$ e a relação entre volume transacionado e volatilidade no mercado.

\section{Análise da variação}

Diversos pesquisadores buscaram estudar a relação entre variações de preços e volume negociado $(\Delta \mathrm{p}$ e $\mathrm{V})$, principalmente para responder se a relação seria significativa. Na Tabela I são resumidos alguns dos principais trabalhos.

Nas relações apresentadas na expressão I, Karpoff (I 987) exibe a proposição de

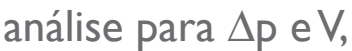

$$
\left\{\begin{array}{c}
V^{-}=h(\Delta p \mid \Delta p \leq 0) \\
V^{+}=h(\Delta p \mid \Delta p>0) \\
E\left(V^{+} \mid \Delta p\right)>E\left(V^{-} \mid \Delta p\right)
\end{array},\right.
$$

em que, é a relação preço-volume negativa dada uma variação negativa nos preços e é a relação preço-volume positiva dada uma variação positiva nos preços. Mediante a expressão proposta por Karpoff (1987), há uma relação assimétrica entre preço e volume, sendo a correlação entre preço e volume maior quando existem variações positivas nos preços. Pela observação da Tabela I, onze dos artigos analisados demonstraram evidências para a existência de relação significativa.

TABELA 1 - Análises variação $\Delta p$ e V

\begin{tabular}{|c|c|c|c|c|}
\hline Autores & Amostra & Período & Intervalo & Relação $\Delta p$ e V \\
\hline $\begin{array}{c}\text { Granger e Morgenstern } \\
\qquad(1963)\end{array}$ & $\begin{array}{c}\text { Mercado de ações agregados, } 2 \\
\text { ações ordinárias }\end{array}$ & $1939-61$ & semanalmente & Não sig. \\
\hline $\begin{array}{c}\text { Godfrey, Granger e Morgens- } \\
\text { tern (1964) }\end{array}$ & $\begin{array}{c}\text { Mercado de ações agregados, } 3 \\
\text { ações ordinárias }\end{array}$ & $\begin{array}{c}1959-62,1951- \\
53,63\end{array}$ & $\begin{array}{c}\text { semanalmente, } \\
\text { diariamente, por } \\
\text { negócios }\end{array}$ & Não sig. \\
\hline Ying (1966) & Mercado de ações agregados & $1957-62$ & diariamente & Sig. \\
\hline Epps (1975) & 20 NYSE bonds & Jan., 1971 & por negócios & Sig. \\
\hline James e Edmister (1983) & 500 ações ordinárias & $1975,77-79$ & diariamente & Não sig. \\
\hline Smirlock e Starks (1985) & 131 ações ordinárias & 1981 & por negócios & Sig. \\
\hline Wood, Mclnish e Ord (1985) & $\begin{array}{c}946 \text { ações ordinárias, } 1138 \text { ações } \\
\text { ordinárias }\end{array}$ & 1971-72, 1982 & minutos & Não sig. \\
\hline Harris (1986) & 479 ações ordinárias & 1976-77 & diariamente & Sig. \\
\hline Medeiros e Doornik (2008) & Índice Bovespa & $2000-2005$ & diariamente & Sig. \\
\hline Araújo e Montini (2013) & Índice Bovespa & $2009-2013$ & diariamente & Não sig. \\
\hline
\end{tabular}

Fonte: Elaborado pelos autores, baseado em Karpoff (1987) 
Visando explicar por que a relação entre $\Delta p$ e $V$ foi significativa, Epps (1975) baseia seu argumento no comportamento dos investidores sob condições específicas de mercado. No caso de Jennings, Starks e Fellingham (198I), os autores buscam explicar a relação por meio da análise do comportamento de investidores desinformados.

Diante disso Epps (1975) argumenta que, nos momentos em que maiores volumes financeiros são transacionados, variações positivas de preço tendem a ocorrer em maior frequência. Para os autores Jennings, Starks e Fellingham (198I), investidores desinformados tendem a negociar menor volume financeiro quando recebem notícias pessimistas. Quando recebem notícias otimistas, no entanto, transacionam maior volume financeiro.

O artigo de Medeiros e Doornik (2008) analisou a relação entre variação de preços e volume transacionado no Brasil no período entre os anos de 2000 e 2005; os autores testaram um modelo proposto por Lee e Rui (2002), exibido no sistema expresso em (2),

$$
\left\{\begin{array}{c}
R_{t}=\alpha_{0}+\alpha_{1} V_{t}+\alpha_{2} V_{t-1}+\alpha_{3} R_{t-1}+u_{t} \\
V_{t}=\beta_{0}+\beta_{1} R_{t}+\beta_{2} V_{t-1}+\beta_{3} V_{t-2}+\varepsilon_{t}
\end{array}\right.
$$

Os autores demonstraram relações significativas entre os coeficientes,, e.Diante disto foi possível evidenciar, no período estudado, a existência de relação contemporânea entre variação dos preços e volume, bem como outra relação defasada para os retornos (variações dos preços). Além disso, os autores exibem a existência de dependência temporal entre as defasagens de 1 e 2 períodos para o volume. Deste modo, Medeiros e Doornik (2008) encontraram para $\circ$ Brasil resultados semelhan- tes ao da maioria dos pesquisadores listados na Tabela I.

O artigo de Araújo e Montini (20I3) incluiu a variável número de negociações () na expressão 2, e buscou comparar as relações nos períodos antes e depois do início das operações por HFT. Pelos resultados, observou-se uma relação negativa e significativa com o volume no período antes do HFT, e uma relação positiva e não significativa pós HFT. Os autores atribuíram o resultado como um possível sinal fraco para maior utilização em andamento do HFT no Brasil, apontando para a demanda de maior número de agentes transacionando no mercado brasileiro.

\section{Análise da volatilidade}

O fluxo de informação nos mercados financeiros é de vital relevância na explicação da volatilidade. Conforme apresenta Karpoff (1987), quanto mais volátil for o fluxo de informação, mais significativa tende ser a relação entre retornos dos preços e volume. Diante disso, a atividade de negociação pode ser uma fonte de explicação para a persistência na volatilidade dos retornos.

Conforme citam Medeiros e Doornik (2008), a persistência no modelo de volatilidade é uma medida de permanência de impacto de um choque sobre a volatilidade; esta análise foi motivada pelos artigos que trataram sobre a hipótese de distribuição mista (Mixture of Distribution Hypothesis - MDH).

Medeiros e Doornik (2008) apontam que os artigos de Clark (1973) e Epps e Epps (1976) foram os primeiros a formalizar a teoria acerca da distribuição dos retornos nos mercados especulativos por meio da $\mathrm{MDH}$. Conforme apresentam Clark (1973) 
e Epps e Epps (1976), os retornos são gerados por distribuições mistas de probabilidade em que o número de chegada de informações é representado por uma série estocástica mista.

Deste modo, a série de retornos é um processo estocástico condicional ao fluxo de informações e as inovações nesse fluxo estão relacionadas com a volatilidade dos retornos. Dado que o fluxo de informações é uma variável não observável, o volume transacionado é utilizado como variável proxy, pois presume-se que as variações no volume são causadas pela chegada de novas informações.

A hipótese de distribuição mista é, portanto, um modelo que explica o processo de formação dos preços nos mercados financeiros. Tal modelo implica dependência estocástica entre volume transacionado e volatilidade. Diversos autores estudaram o modelo - na Tabela 2 resume-se os principais trabalhos.

O processo de desenvolvimento dessa teoria começa no artigo de Clark (1973); conforme o autor, por meio de um processo estocástico, um novo processo subordinado denominado pode ser modelado. Deste modo, pode ser percebido como o processo de evolução dos preços e é uma medida de tempo que denota a velocidade da evolução do processo; o volume transacionado seria a variável para representar a velocidade de tal evolução.

Mediante o modelo apresentado, Clark (1973) postula que a volatilidade dos preços nos mercados especulativos é explicada por uma função simples do volume. Por meio de uma série de preços do mercado futuro de algodão, o autor estuda a relação e encontra uma associação positiva e significativa.
O artigo de Epps e Epps (1976) apresenta evidências empíricas adicionais ao artigo de Clark (1973), demonstrando a variância condicional como função do volume transacionado.

O modelo apresentado em Epps e Epps (1976), por sua vez, é baseado nas expectativas dos investidores. Segundo os autores, se em dado momento " $t$ " a expectativa do trader é diferente do momento " $t$ - I", o preço no instante " $t$ - I" possuía excesso de oferta ou demanda. Os autores citam que esse desequilíbrio pode ter ocorrido pela chegada de novas informações ou pela discrepância entre o preço de mercado e o chamado preço "nulo", um preço adotado quando o mercado não possui excesso de oferta/demanda.

Dado que novas informações afetam os preços "nulos" dos diferentes traders, o comportamento destes direciona o preço em " $t$ - I" ao novo preço " $t$ ". $O$ existente excesso de oferta/demanda é, assim, equilibrado, fazendo com que os preços de mercado e "nulos" tornem-se iguais.

Em mercados em que novas informações alteram o comportamento dos investidores como os supracitados, diversos momentos de opiniões discrepantes ocorrem. Os investidores que possuem preços "nulos" acima do preço de equilíbrio no momento " $t$ " irão comprar as ações dos investidores que possuírem preço "nulo" abaixo do preço de equilíbrio no momento " $t$ ".

Diante disto, Epps e Epps (1976) definem $\mathrm{MDH}$ utilizando o volume transacionado como medida para o comportamento discrepante entre os traders quando eles revisam seus preços devido à chegada de novas informações no mercado. Quanto maior o desacordo, maior o volume transa- 
cionado; desse modo, os autores sugerem uma relação positiva entre volatilidade e volume transacionado. No artigo, os dados confirmam a relação esperada.

Conforme apresentam Tauchen e Pitts (1983), os artigos de Clark (1973) e Epps e Epps (1976) são complementares, dado que ambos demonstram pontos relevantes para entendimento dos mercados especulativos. Entretanto, os autores demonstram que esses modelos funcionam para mercados maduros; para mercados em crescimento, os modelos apresentam inconsistências.

Diante disto, Tauchen e Pitts (1983) analisam o MDH para o caso de mercado em crescimento utilizando como exemplo o mercado futuro de títulos de dívida dos E.U.A. (T-Bill). Como resultado, os autores demonstraram que o volume médio transacionado cresce linearmente com o número de agentes no mercado. No caso da volatilidade, a presença de mais agentes tende a causar decréscimo na variância dos preços. Porém, devido à presença de mais agentes, o grau de desacordo entre eles pode aumentar, fazendo com que a variância dos preços possua relação positiva com o volume transacionado.

O artigo de Lamoureux e Lastrapes (1990) explora o tema, levando em consideração que os incrementos da variância dos preços são heterocedásticos; a relação da série com a taxa de chegada de informações diária é positiva. Para testar essa relação, os autores estimam um modelo Autoregressivo com Heterocedasticidade Condicional Generalizado de primeira ordem - GARCH(I,I) - utilizando o volume para representar o fluxo de informações.

Os autores testaram o comportamento dos efeitos GARCH (Generalized Autore- gressive Conditional Heteroskedasticity) com e sem a presença da variável de volume transacionado. $O$ artigo de Lamoureux e Lastrapes (1990) encontrou resultados relevantes, demonstrando que os efeitos $\mathrm{GARCH}$ tendem a desaparecer na presença da variável de volume no modelo. Dessa forma, os autores motivam a utilização dos modelos GARCH nos estudos sobre comportamento dos preços de ativos financeiros.

O artigo de Andersen (1996) apresenta contribuições significativas devido à adição de novos elementos à hipótese de distribuição mista dos retornos nos mercados especulativos. $O$ autor desenvolve um modelo que analisa a relação retornovolume integrando a perspectiva da volatilidade estocástica. Desta forma, estuda uma versão modificada do modelo $\mathrm{MDH}$ (Modified MDH).

Ainda conforme Andersen (1996), as principais diferenças em relação ao modelo original são: a presença de uma variável que considera o componente de liquidez nas negociações e a suposição de que 0 processo de chegada de informações segue uma distribuição de Poisson, e não uma distribuição Normal, tal qual proposta em Epps e Epps (1976).

Diante disso, o fluxo de informações é presumido para representar um processo de volatilidade estocástica que direciona retornos e volume. Segundo os resultados de Andersen (1996), a versão modificada do modelo $\mathrm{MDH}$ apresentou uma especificação que se ajustou aos dados, além de uma melhor performance de análise em relação ao modelo original.

O artigo de Lee e Rui (2002) analisou a relação entre retornos, volume e volatilidade buscando superar uma limitação 
observada nos demais estudos: a análise de somente um mercado. No trabalho de Lee e Rui (2002) os autores buscaram comparar a relação entre mercados de países diferentes (Nova lorque, Tóquio e Londres); os autores demonstraram que a relação entre volatilidade e volume transacionado permaneceu significativa mesmo se analisada em mercados diferentes.

No artigo de Medeiros e Doornik (2008), os autores analisaram a relação entre volatilidade e volume no mercado brasileiro.As análises ocorreram por meio do ajuste do modelo apresentado na expressão 3:

$$
\left\{\begin{array}{c}
R_{t}=\alpha_{0}+\alpha_{1} R_{t-1}+\varepsilon_{t} \\
\sigma_{t}^{2}=\beta_{0}+\beta_{1} \varepsilon_{t-1}^{2}+\beta_{2} \sigma_{t-1}^{2}+\beta_{3} V_{t}
\end{array}\right.
$$

Nesta, o processo estocástico dos retornos () foi expresso por um termo autorregressivo e a equação da variância condicional foi especificada por um GARCH(I,I), com um dos termos sendo o volume transacionado (). $O$ termo de erro possui comportamento ; a soma dos parâmetros é a medida de persistência na variância do termo .

Ressalta-se que a persistência é uma medida de permanência de impacto de um choque sobre a volatilidade do preço de um ativo; caso as condições de estabilidade do modelo sejam aceitas, os valores da persistência variam entre 0 e I. Quanto mais próximo de I, maior o tempo que um choque na série levará para se dissipar.

Quanto à relação entre volatilidade e volume, a pesquisa de Medeiros e Doornik (2008) apresentou resultado similar ao dos demais autores quando o sinal da relação foi observado, porém a significância foi diferente. Desta forma, a relação entre volatilidade e volume foi positiva, porém não significante.

No caso da interpretação da persistência, os autores encontraram resultados similares aos de Lamoureux e Lastrapes (1990), em que os termos GARCH ( e ) tenderam a diminuir na presença da variável de volume. Entretanto, diferente de Lamoureux e Lastrapes (1990) - em que os coeficientes não foram significativos -, nas estimações de Medeiros e Doornik (2008) os efeitos da persistência se mantiveram significativos. Diante destes resultados, os autores concluem que para o Brasil o modelo $\mathrm{MDH}$ apresentou fraco ajuste no período estudado.

TABELA 2 - Análises relação Volatilidade e Volume

\begin{tabular}{|c|c|c|c|c|}
\hline Autores & Amostra & Período & Intervalo & $\begin{array}{l}\text { Relação Volat. } \\
\text { x Volume }\end{array}$ \\
\hline Clark (1973) & Mercado Futuro de Algodão & 1947-1955 & diário & Sig. \\
\hline Epps e Epps (1976) & Ações da bolsa de Nova lorque & 1971 & diário & Sig. \\
\hline Tauchen e Pitts (1983) & $\begin{array}{l}\text { Mercado futuro títulos de dívida E.U.A. } \\
\text { (T-Bill) }\end{array}$ & 1976-1979 & diário & Sig. \\
\hline $\begin{array}{l}\text { Lamoureux e Lastrapes } \\
\qquad(1990)\end{array}$ & Ações da bolsa de Nova lorque & $1980-1983$ & diário & Sig. \\
\hline Andersen (1996) & Ações da bolsa de Nova lorque & 1973-1991 & diário & Sig. \\
\hline Lee e Rui (2002) & $\begin{array}{c}\text { Índices das bolsas de Nova lorque, } \\
\text { Tóquio e Londres }\end{array}$ & $1973-1999$ & diário & Sig. \\
\hline Medeiros e Doornik (2008) & Índice Bovespa & $2000-2005$ & diário & Não sig. \\
\hline
\end{tabular}

Fonte: Elaborado pelos autores 


\section{METODOLOGIA Dados e variáveis}

A pesquisa no Brasil de Medeiros e Doornik (2008) abarcou o período entre os anos 2000 e 2005. Na presente pesquisa, as estimações para o período antes HFT correspondem ao período utilizado por Medeiros e Doornik (2008) e o pós HFT, entre os anos 2009 e 2014.

Esta operacionalização foi importante para comparar os resultados no mesmo período e avaliar a existência de possíveis alterações. O período entre 2006 e 2008 não foi incorporado às análises por ter sido o período da crise financeira, portanto capaz de causar algum viés nas estimações dos modelos.

Os efeitos do número de negociações foram incorporados ao modelo.A principal justificativa para considerar essa variável está relacionada à rápida realocação de capital referenciada pelo HFT; deste modo, muitas negociações ocorrem em milésimos de segundos, causando um aumento significativo do número de negociações na bolsa.

O sistema estimado para verificar estas relações é apresentado na expressão 4: o modelo apresenta algumas modificações em relação aos sistemas estimados em Medeiros e Doornik (2008).

Quanto à análise entre volatilidade e volume negociado, o mesmo sistema de equações utilizado em Medeiros e Doornik (2008) foi estimado (expressão 5); no artigo dos autores a relação demonstrou ser não-significativa. Contudo, conforme apresentam autores como Tauchen e Pitts (1983) e Lamoureux e Lastrapes (1990), o contrário deveria ocorrer.

Os dados de Retornos, Volume e Número de Negociações foram obtidos na plataforma de dados financeiros ECONO-
MATICA. O procedimento metodológico adotado em Medeiros e Doornik (2008) também foi realizado: os valores das séries foram deflacionados pelo IGP-DI e transformados em índice (base 100 para 03.01.2000). A análise de dados foi viabilizada pelo software EViews 7.

$$
\begin{gathered}
\left\{\begin{array}{c}
R_{t}=\alpha_{0}+\alpha_{1} V_{t}+\alpha_{3} R_{t-1}+u_{t}, \\
V_{t}=\beta_{0}+\beta_{1} R_{t}+\beta_{4} N_{t}+\varepsilon_{t}, \\
N_{t}=\gamma_{0}+\gamma_{1} N_{t-1}+\eta_{t} .
\end{array}\right. \\
\left\{\begin{array}{c}
R_{t}=\alpha_{0}+\alpha_{1} R_{t-1}+\varepsilon_{t}, \\
\sigma_{t}^{2}=\beta_{0}+\beta_{1} \varepsilon_{t-1}^{2}+\beta_{2} \sigma_{t-1}^{2}+\beta_{3} V_{t} .
\end{array}\right.
\end{gathered}
$$

A variável é representada como o número de negociações; espera-se um aumento nesta variável no período de presença do HFT.

Séries econômicas possuem comportamento não-estacionário, dado possuírem tendência estocástica e pelo menos uma raiz unitária. Tal comportamento ocorre devido à proximidade dos processos geradores a passeios aleatórios (random walks). $\bigcirc$ artigo de Phillips (1986) demonstra que uma análise de regressão com séries desse tipo apresenta o problema da regressão espúria, não servindo para estimações financeiras e econômicas.

Para verificar a estacionariedade das séries foram utilizados os testes de raiz unitária. No presente artigo, dois deles são apresentados:Teste de Dickey e Pantulla (DP) e Teste de Dickey-Fuller Aumentado (ADF).

O sistema expresso em 4 foi estimado pelo método da máxima verossimilhança com informação completa (MV), este método foi utilizado para obter resultados que pudessem ser comparados com os artigos de Medeiros e Doornik (2008) e Araújo e Montini (20I3). O presente artigo 
complementou essas análises por estimar o sistema 4 por regressões em três estágios (Three-Stage Least Squares - 3SLS) e por método dos momentos generalizados (Generalized Method of Moments - GMM).

Desse modo, esses métodos possibilitaram verificar problemas de endogeneidade e heteroscedasticidade, além de permitir a estimativa de forma a corrigir tais problemas. $O$ sistema expresso em 5 foi estimado por um GARCH $(I, I)$ adotando para otimização o método de Marquadt. Este foi escolhido por possibilitar a comparação com o artigo de Medeiros e Doornik (2008).

\section{ANÁLISE DOS DADOS}

Nesta etapa são discutidas as análises delineadas durante a metodologia, isto é, a divisão dos períodos antes e depois da eminência do HFT, realização dos testes de raiz unitária e estimação dos modelos propostos.

\section{Testes de raiz unitária}

Para verificar a estacionariedade das séries, foram utilizados 2 testes: o teste de Dickey e Pantulla, utilizado para avaliar a existência de 2 raízes unitárias contra somente I raiz unitária e o teste ADF, utilizado para verificar a existência de I raiz unitária contra 0 raiz unitária (ausência de raiz unitária). Os testes para verificar a estacionariedade das séries foram resumidos nas Tabelas 3 e 4.

Como resultados, nos testes de Dickey-Pantulla foram rejeitadas as hipóteses de existência de 2 raízes unitárias. Nos testes ADF, foram identificadas evidências para rejeitar a hipótese de existência de I raiz unitária. Desse modo, não houve necessidade de transformação nos dados para obtenção da estacionariedade.

\section{Estimação dos modelos}

Após a constatação de que as séries são estacionárias, buscou-se estimar os modelos. Nas Tabelas 5 e 6 são apresentadas as estimações. Na primeira coluna são apresentados os parâmetros relacionados às variáveis estudadas; nas demais colunas são comparados os resultados das pesquisas realizadas por Medeiros e Doornik (2008), Araújo e Montini (20I3) e do presente artigo.

A análise da relação entre retornos, volume e negociações foi possibilitada pela observação do sistema expresso em 4, detalhado na Tabela 5. Para cada período, foram apresentados os resultados com as estimações dos modelos MV, 3SLS e GMM, além disto, as estimações observadas nos artigos de Medeiros e Doonik (2008) e Araújo e Montini (20|3).

TABELA 3 - testes raiz unitária (03.01.2000 - 29.12.2005)

\begin{tabular}{c|c|c|c|c|c|c}
\hline Variáveis & Teste DP & $\boldsymbol{t}$ & Resultado & Teste ADF & $\boldsymbol{t}$ & Resultado \\
\hline & sem tend. e const. & $-12,362$ & não existem 2 ru & com const. & $-18,188$ & não existe 1 ru \\
\hline & sem tend. e const. & $-12,883$ & não existem 2 ru & com const. & $-14,191$ & não existe 1 ru \\
\hline & sem tend. e const. & $-16,640$ & não existem 2 ru & com const. & $-18,712$ & não existe 1 ru \\
\hline
\end{tabular}

TABELA 4 - testes raiz unitária (02.01.2009 - 07.07.2014)

\begin{tabular}{c|c|c|c|c|c|c}
\hline Variáveis & Teste DP & $\boldsymbol{t}$ & Resultado & Teste ADF & $\boldsymbol{t}$ & Resultado \\
\hline & sem tend. e const. & $-15,678$ & não existem 2 ru & com const. & $-7,400$ & não existe 1 ru \\
\hline & sem tend. e const. & $-14,256$ & não existem 2 ru & com const. & $-9,664$ & não existe 1 ru \\
\hline & sem tend. e const. & $-16,333$ & não existem 2 ru & com const. & $-16,698$ & não existe 1 ru \\
\hline
\end{tabular}


Por meio do teste de Breusch-Pagan, verificou-se a existência de heteroscedasticidade nas estimações por 3SLS e MV; o teste foi realizado para cada equação separadamente, e os resultados evidenciaram o problema da heteroscedasticidade. Quanto à identificação do modelo, o teste de Hansen-Sargan evidenciou que os modelos estavam identificados, não existindo problemas de endogeneidade.

Para corrigir o problema da heteroscedasticidade, o sistema foi estimado por GMM com matriz de ponderação robusta. Diante disso, as estimativas para a expressão 4 foram eficientes e consistentes. Em relação à interpretação dos sinais, os modelos apresentaram as mesmas informações.

Ao comparar as estimativas, percebe-se que as relações contemporâneas retorno e volume apresentaram mesmo sinal, porém significâncias diferentes. Ou seja, quando a variável "quantidade de negociações" foi imposta, observou-se uma relação contemporânea positiva entre variação dos preços e volume transacionado - esta não foi, entretanto, significativa. No período pós HFT, a relação entre as variáveis se inverteu, mantendo-se não significativa. Isto ocorreu pelo fato de que no período o volume na bolsa se manteve estagnado e os retornos diminuíram, conforme apresenta Rocha (20/3).

Desse modo, o presente artigo demonstrou não existir uma relação significativa entre variação dos preços e volume negociado nos períodos estudados. Enquadrase, portanto, com os resultados apresentados em Granger e Morgenstern (1963), Godfrey, Granger e Morgenstern (1964), James e Edmister (1983), Wood, Mclnish e Ord (1985) e Araújo e Montini (2013).

Quanto à análise entre número de negociações e volume, o presente artigo apresentou resultados diferenciados em relação ao trabalho de Araújo e Montini (20|3); graças à revisão do modelo, as relações entre as variáveis se tornaram mais coerentes com a teoria.

O coeficiente comparado em ambos os períodos demonstra o crescimento do número de negociações () com o passar dos anos, principalmente na presença das transações de alta frequência. Tanto no período antes HFT quanto no pós HFT a relação foi positiva e significante, existindo um aumento no coeficiente do período pós HFT. Isto demonstra que o número de negociações influencia o volume transacionado. $O$ crescimento das operações HFT, contudo, garante mais força a tal influência.

Na Tabela 6 são apresentadas as estimativas que analisaram a relação entre volatilidade e volume, exibidas na expressão $5 . \mathrm{Na}$ primeira coluna são visualizados os parâmetros relacionados às variáveis; nas colunas 2 e 3 podem ser observados os resultados do artigo de Medeiros e Doornik (2008) com os sistemas sem e com a variável volume, respectivamente. Nas colunas 4 e 5 , as estimativas do período antes HFT, sem e com a variável volume; e nas colunas 6 e 7 os resultados para o período pós HFT sem conter e contendo a variável volume.

Conforme comentado, o artigo de Medeiros e Doornik (2008), contrariando demais autores, demonstrou não existir relação significativa entre volatilidade e volume. No presente artigo, demonstra-se que a relação entre volatilidade e volume é positiva e significativa no mercado brasileiro; os resultados estão de acordo com os demais pesquisadores da área.

Verificou-se que, conforme apresentou Karpoff (1987), a força da relação tendeu a aumentar à medida que o número de 
TABELA 5 - Estimações Retorno x Volume (expressão 4)

\begin{tabular}{|c|c|c|c|c|c|c|}
\hline Par. & $\begin{array}{c}\text { Medeiros } \\
\text { e Doornik } \\
(2008)\end{array}$ & $\begin{array}{c}\text { 3SLS - MV } \\
(2000-2005)\end{array}$ & $\begin{array}{c}\text { GMM } \\
(2000-2005)\end{array}$ & $\begin{array}{c}\text { Araujo e Montini } \\
\text { (2013) }\end{array}$ & $\begin{array}{c}\text { 3SLS - MV } \\
(2009-2014)\end{array}$ & $\begin{array}{c}\text { GMM } \\
(2009-2014)\end{array}$ \\
\hline & $-0,031^{* * *}$ & $-0,006$ & $-0,006$ & $-0,0011$ & 0,004 & 0,0044 \\
\hline & $0,001^{\text {*** }}$ & 0,0001 & 0,000063 & 0,0001 & $-0,00004$ & $-0,000039$ \\
\hline & $-0,453^{* *}$ & $-0,467^{* * *}$ & $-0,4669^{* * *}$ & $-0,516^{* * *}$ & $-0,511^{* * *}$ & $-0,5149^{* * *}$ \\
\hline & $155,502^{* \star *}$ & 0,944 & 1,303 & $154,506^{\star * *}$ & $-11,909$ & $-12,3876$ \\
\hline & 144,275 & 4,292 & 1,4958 & 94,200 & $-3,515$ & $-9,16391$ \\
\hline & $-0,292^{* * *}$ & - & - & $-0,332^{* * *}$ & - & - \\
\hline & $-0,172^{* * *}$ & - & - & $-0,022$ & - & - \\
\hline & - & $1,010^{\star * *}$ & $1,00613^{* * *}$ & $-0,124$ & $1,136^{* * *}$ & $1,141152^{* * *}$ \\
\hline & - & $130,213^{* * *}$ & $130,549^{* * *}$ & $134,519^{* * *}$ & $136,047^{\star * *}$ & $134,4609^{* * \star}$ \\
\hline & - & $-0,250^{* * *}$ & $-0,25238^{* * *}$ & $-0,310^{* * *}$ & $-0,324^{* * *}$ & $-0,310^{* * *}$ \\
\hline & - & - & - & 92,204 & - & - \\
\hline \multicolumn{2}{|c|}{ Teste B.P. ${ }^{1}$} & $\begin{array}{l}\text { Eq-Ret } \\
\text { Eq-Vol: } \\
\text { Eq-Neg: }\end{array}$ & $\begin{array}{l}0,19) \\
0,00) \\
(0,00)\end{array}$ & - & $\begin{array}{l}\text { Eq-Ret: } \\
\text { Eq-Vol: } \\
\text { Eq-Neg: }\end{array}$ & $\begin{array}{l}2(0,001) \\
2(0,000) \\
9(0,000)\end{array}$ \\
\hline \multicolumn{2}{|c|}{ Teste H.S. ${ }^{2}$} & \multicolumn{2}{|c|}{$0,030(0,863)$} & - & \multicolumn{2}{|c|}{$3,201(0,074)$} \\
\hline
\end{tabular}

${ }^{1}$ Teste Breusch-Pagan (Heteroscedasticidade), 2Teste Hansen-Sargan (Superidentificação)

${ }^{* *}$ Significante a $5 \% .{ }^{* * *}$ Significante a $1 \%$.

TABELA 6 - Estimações Volatilidade x Volume (expressão 5)

\begin{tabular}{|c|c|c|c|c|c|c|}
\hline P. & $\begin{array}{l}\text { M. e D. } \\
(2008)^{1}\end{array}$ & M. e D. (2008) & $\begin{array}{l}\text { Exp. } 5 \text { s/ vol. } \\
(2000 \text { - 2005) }\end{array}$ & $\begin{array}{l}\text { Exp. } 5 \text { c/ vol. } \\
(2000 \text { - 2005) }\end{array}$ & $\begin{array}{l}\text { Exp. } 5 \text { s/ vol. } \\
(2009 \text { - 2014) }\end{array}$ & $\begin{array}{l}\text { Exp. } 5 \mathrm{c} / \mathrm{vol} \\
(2009-2014)\end{array}$ \\
\hline & 0,000179 & $-0,000122$ & 0,000648 & 0,000557 & 0,000535 & 0,000535 \\
\hline & $-0,478^{\star * *}$ & $-0,475^{\star \star \star}$ & $-0,482^{* \star *}$ & $-0,482^{* \star *}$ & $-0,497^{\star \star \star *}$ & $-0,490^{\star * \star}$ \\
\hline & 0,000032 & 0,0000046 & $0,000031^{* * *}$ & $-0,000059$ & $0,0000182^{* * *}$ & $-0,000145^{* * *}$ \\
\hline & $0,104^{* * *}$ & $0,164^{* * *}$ & $0,101^{* * *}$ & $0,105^{\star * *}$ & $0,109^{* * *}$ & $0,122^{* * *}$ \\
\hline & $0,836^{\star \star \star *}$ & $0,703^{* * *}$ & $0,842^{* \star *}$ & $0,835^{\star * *}$ & $0,835^{\star \star *}$ & $0,810^{* * *}$ \\
\hline & - & 0,0000006 & - & $0,000000866^{* *}$ & - & $0,0000016^{* * *}$ \\
\hline Per. & 0,940 & 0,867 & 0,943 & 0,939 & 0,944 & 0,932 \\
\hline
\end{tabular}

1Medeiros e Doornik (2008). ${ }^{*}$ Significante a $5 \%$. *** Significante a $1 \%$.

negociações aumentou. Pela comparação dos valores e nos períodos antes HFT e pós HFT pode-se comprovar esta citação. Cabe ressaltar que na Tabela 5 constatouse $\circ$ aumento do número de negociações no período pós HFT.

Os resultados também foram compatíveis com o artigo de Lamoureux e Lastrapes (1990). Deste modo, os efeitos GAR$\mathrm{CH}$, medidos pelos coeficientes e tiveram seus valores reduzidos quando a variável volume esteve presente na estimativa. Por meio deste resultado, observa-se também que a persistência () tendeu a diminuir na presença da variável volume, também em consonância a Lamoureux e Lastrapes (1990) e Medeiros e Doornik (2008).

Dado que os algoritmos de alta frequência conseguem realizar operações em altíssima velocidade e com volumes significativos, espera-se que algum choque na série financeira seja dissipado em menos tempo. $O$ valor da persistência no período pós HFT foi levemente menor que em relação ao período antes HFT (antes $=0,939$ $>$ pós $=0,932$ ), porém ainda não é possível 
afirmar que num ambiente com operações HFT os impactos dos choques nas séries tenham menor permanência.

\section{CONSIDERAÇÕES FINAIS}

A utilização das operações por alta frequência no Brasil é recente. Enquanto nos E.U.A. o HFT passa por um momento de desconfiança, com dúvidas até mesmo sobre a competência de seus operadores, no Brasil os executivos desejam aumentar o número de operações por acreditar que o país está em estágio semelhante ao dos E.U.A. antes do rápido crescimento no passado.

Conforme Aldridge (20I3), o objeto é particularmente complexo por se tratar de um campo interdisciplinar em que duas áreas complexas de estudos devem ser dominadas: finanças em alta frequência e ciências da computação. Deste modo, profissionais da área precisam de capacitação - seja para entender finanças ou dominar programação.Apesar da demanda significativa em busca de informações, no entanto, pouco ainda é publicado.

Diante disso, o artigo de Araújo e Montini (20I3) contribuiu ao analisar a relação entre retornos, volume e número de negociações no mercado financeiro, dividindo períodos antes e depois do início do HFT no Brasil. A relação destas variáveis com a volatilidade não foi verificada.

Uma das hipóteses levantadas pelos oposicionistas ao HFT, conforme Kirilenko et al (20l I), seria que os investidores de HFT seriam capazes manipular o mercado em decorrência da rápida reação dos algoritmos a novas informações e altíssimo volume de transação financeira. Este comportamento poderia gerar o acionamento descontrolado de ordens start (entrada) ou stop (saída), alterando o equilíbrio do mercado e aumentando a volatilidade.

Em busca de estudar tais afirmações, o presente artigo teve por objetivo analisar a relação entre volatilidade e volume na bolsa de valores brasileira em momentos antes e pós-iniciação das estratégias HFT. Deste modo, o artigo revisou um dos modelos de retorno e volume estudado em Araújo e Montini (20/3) e atualizou as estimativas com dados mais recentes.

Quanto à relação entre preços e retornos, o artigo demonstrou não existir uma relação significativa nos períodos estudados; resultados similares foram demonstrados com os trabalhos de Granger e Morgenstern (1963), Godfrey, Granger e Morgenstern (1964), James e Edmister (1983), Wood, Mclnish e Ord (1985) e Araújo e Montini (20I3).

Quanto à análise entre número de negociações e volume, atingiram-se resultados demonstrativos do crescimento do número de negociações com o passar dos anos, principalmente na presença do HFT. Tanto no período antes HFT quanto no pós HFT a relação foi positiva e significativa. Desta forma, o número de negociações influencia o volume transacionado; com o crescimento das operações HFT, essa influência apresentou ainda mais força.

Quanto à relação entre volatilidade e volume, ao contrário dos resultados apresentados em Medeiros e Doornik (2008), os coeficientes foram positivos e significativos, sendo que a força da relação tendeu a aumentar à medida que o número de negociações aumentou. $O$ resultado encontrado ocorreu conforme os modelos estudados em Tauchen e Pitts (1983), Karpoff (1987) e Lamoureux e Lastrapes (1990).

Desse modo, o presente artigo con- 
tribuiu de forma relevante ao demonstrar que a hipótese de distribuição mista (Mixture of Distribution Hypothesis - MDH) está adequada ao mercado brasileiro, sendo ainda mais presente nesta nova fase do mercado, com as operações em alta frequência.

Este resultado traz um alerta aos operadores de mercado: o uso exagerado do HFT pode gerar $\mathrm{o}$ acionamento de forma descontrolada de ordens start (entrada) ou stop (saída), alterando o equilíbrio do mercado brasileiro num curto espaço de tempo.

Limitamo-nos a analisar somente a série do índice Bovespa, sendo necessário em trabalhos futuros verificar as relações numa gama maior de ativos, tais como índices de dividendos, small caps e energia elétrica. A metodologia de divisão de períodos em antes e pós HFT também é um fator limitante, dado que no período pós HFT o número de negociações pode ter aumentado não somente pela maior utilização de algoritmos, mas também devido à maior utilização dos home brokers por pessoa física.

Este fator não invalida os resultados, dado que muitas dessas plataformas possuem algoritmos implementados e estão sendo adotadas por investidores/operadores. Diante disso, em trabalhos futuros torna-se necessário investigar o impacto específico de uma operação HFT no mercado: isto será possível com a disponibilização de bancos de dados contendo informações evento a evento (tick by tick). 


\section{REFERÊNCIAS}

ALDRIDGE, I. High-Frequency trading: a practical guide to algorithmic strategies and trading systems. New Jersey: John Wiley \& Sons, 2010.

ALDRIDGE, I. High-Frequency trading: a practical guide to algorithmic strategies and trading systems. 2nd. New Jersey: John Wiley \& Sons, 2013.

ANDERSEN,T. G. Return volatility and trading volume: An information flow interpretation of stochastic volatility. The Journal of Finance, v. 5I, n. 0I, p.p. 169-204, 1996.

ARAÚJO, A. C.; MONTINI, A. A. High Frequency Trading: Abordagem Clássica para Análise de Preço-Volume em uma Nova Microestrutura de Mercado. In: SEMINÁRIOS EM ADMINISTRAÇÃO - SEMEAD, 16., 20।3, São Paulo, Anais... São Paulo: SEMEAD, 2013.

CLARK, P. K.. A subordinated stochastic process model with finite variance for speculative prices. Econometrica, v. 4I, n. 0I, P.p. I35-I55, 1973.

EPPS, T.W. Security price changes and transaction volumes: theory and evidence. American Economic Review, v. 65, n. 0I, p.p. 586-597, 1975.

EPPS, T. W. The demand for brokers' services: the relation between security trading volume and transaction cost. Bell Journal of Economics, v. 7, n. I, p.p. 163-194, 1976.

GODFREY, M. D.; GRANGER, C.W. J.; MORGENSTERN, O. Random walk hypothesis of stock market behavior. Kyklos, v. 17, n. 0I, p.p. I-30, 1964.

GRANGER, C. W. J.; MORGENSTERN, O. Spectral analysis of New York stock market prices. Kyklos, v. I6, n. 0I, p.p. I-27, 1963.
HARRIS, L. Cross-Security tests of the mixture of distributions hypothesis. Journal of Financial and Quantitative Analysis, v. 2I, n. I, p.p. 39-46, 1986.

IOSCO. Regulatory issues raised by the impact of technological changes on market integrity and efficiency. relatório final, international organization of securities comissions - IOSCO. 20I2. Disponível em: http://www.iosco.org/library/ pubdocs/pdf/IOSCOPD36I.pdf. Acesso em: 17/04/20I3.

JAMES, C.; EDMISTER, R. O. The relation between common stock returns trading activity and market value. Journal of Finance, v. 38, n. 0I, p.p. 1075-1086, 1983.

JENNINGS, R. H., STARKS, L. T., \& FELLINGHAM, J. C. An equilibrium model of asset trading with sequential information arrival. Journal of Finance, v. 36, n. 이, p.p. I43-I6I, I98I.

KARPOFF, J. M. The relation between price changes and trading volume: a survey. The Journal of Financial and Quantitative Analysis, v. 22, n. 01, p.p. 109-126, 1987.

KIRILENKO,A.A.; KYLE, A. S.; SAMADI, M.;TUZUN, T.The flash crash: the impact of high frequency trading on an electronic market. Working Paper, 20II. Disponível em http://ssrn.com/abstract $=1686004$ or http://dx.doi. org//0.2139/ssrn.1686004. Acesso em 19/0I/2013.

KUPFER, J. P. (20II, Maio 23). Utopias em alta frequencia. ESTADÃO conteúdo. Recuperado em I5 Maio, 20I3, de <http://blogs.estadao.com.br/ jpkupfer/utopias-em-alta-frequencia/?doing_wp_cron $=\mid 363564940$. 6436629295349|21093750>.

LAMOUREUX, C. G.; LASTRAPES, W. D. Heteroskedasticity in stock return data: volume versus GAR$\mathrm{CH}$ effects. Journal of Finance, v. 45, n. 01 , p.p. $221-229$, 1990.

LEE, B. S.; RUI, O. M. The dynamic relationship between stock returns and trading volume: domestic and cross-country evidence. Journal of Banking and Finance, v. 26, n. 0I, p.p. 5I-78, 2002.

MAZZONI, C. High-frequency trading brings American whiz-kid to Brazil. VALOR.com. 2012. Recuperado em I6 Maio, 2013, de <http:// www.valor.com.br/international/ news/2878790/high-frequencytrading-brings-american-whiz-kid -brazil\#ixzz2TV7WJPfO>.

MEDEIROS, O. R.; DOORNIK, B. F. $N$. V. The empirical relationship between stock returns, return volatility and trading volume in the Brazilian stock market. Brazilian Business Review - BBR, v. 5, n. 0I, p.p. 01-17, 2008.

PHILLIPS, P. C. B. Understanding spurious regressions in econometrics. Journal of Econometrics, v. 33, n. 0 I, p.p. 3। I-340, 1986.

PORTNOY, K. High frequency trading and the stock market: a look at the effects of trade volume on stock price changes. The Park Place Economist, v. 19, n. 0I, p.p. 35-47, 2011.

PORTUGAL, M. (2010, Agosto 09). Investidor de alta frequência ganha seu espaço dentro da Bovespa. EXAME.com. Recuperado em: 09 Abril, 20।3, de <http://exame.abril. com.br/mercados/noticias/investidor-alta-frequencia-ganha-seu-espaco-dentro-bovespa-585958>.

ROCHA, A. A. (2012, Abril 0I). estagnação da bolsa: faltam investidores ou cias? VALOR.com. Recuperado em 07 Maio, 20I3, de http:// www.valor.com.br/valor-investe/o-estrategista. 
SEABRA, L. (20I4, Março 10). Ho- SMIRLOCK, M.; STARCKS, L. A mem $\times$ Máquina. VALOR.com. Recuperado em 16 Abril, 2014, de http://www.valor.com.br/financas/3455242/homem-x-maquina\#ixzz2vaN82tBT.

SEC. (20I0). Concept Release on Equity Market Security, 17 CFR Part 242, Release No. 34-6/358, Securities and Exchange Commission. transactional approach to testing information arrival models. Working Paper. 1984.

TAUCHEN, G.; PITTS, M. The price variability-volume relationship on speculative markets. Econometrica, v. 5 I, n. 0 I, p.p. $485-505$, 1983. VUORENMAA, T. A. The good, the bad, and the ugly of automated high-frequency trading. The Journal of Trading, v. 8, n. 01 , p.p. 5874, 2013.

WOOD, R.A.;MCINISH,T.H.;ORD,J.K. An investigation of transactions data for NYSE stocks. Journal of Finance, v. 60, n. 0I, p.p. 723-739, 1985.

YING, C. C. Stock market prices and volumes of sales. Econometrica, v. 34, n. 0I, p.p. 676-686, 1966. 